\title{
Evidence for short-term variability of Jupiter's decimetric emission from VLA observations
}

\author{
D. Santos-Costa ${ }^{1}$, S. J. Bolton ${ }^{1}$, and R. J. Sault ${ }^{2}$ \\ 1 Space Science Department, SwRI, Tx, USA \\ e-mail: daniel.santoscosta@swri.edu \\ 2 University of Melbourne, Australia
}

Received 18 March 2009 / Accepted 21 August 2009

\begin{abstract}
Aims. We present the first evidence of short-term variations of Jupiter's radiation-belt emission obtained with interferometric measurements. Over a two-month period of observational time in 2002, the Jovian synchrotron emission was observed with the Very Large Array (VLA).

Methods. The images constructed at the wavelength of $6 \mathrm{~cm}$ demonstrate significant changes in the spatial structure of the brightness distribution. The comparisons of the two-dimensional maps with another campaign of VLA observations made in May 1997 confirm our discovery of changes in Jupiter's synchrotron emission on a time-scale of days to weeks.

Results. For a series of central meridian longitudes (CMLs), the radiation peak near $1.4 R_{\mathrm{J}}$ was observed to shift back and forth from one side of the planet to the other between October and December 2002. The change in the location of the emission peak was found to be the result of fluctuations in the peak brightness distribution by $10 \%$ up to $40 \%$. These fluctuations are too significant to be associated with the small variation of the geometric parameter $D_{\mathrm{E}}$ (the declination of the Earth as seen from Jupiter) during the campaign of observations. We have demonstrated that the variability of the synchrotron emission peak was observed when the angular sectors covering the Jupiter SIII longitudes $\lambda_{\text {III }}$ where the field strength along the equatorial magnetic surface is maximum or minimum were monitored.

Conclusions. Short-term variations of Jupiter's synchrotron emission are expected to be reported when specific CMLs are observed and changes in the distributions of the $30-40 \mathrm{MeV}$ electron population occur simultaneously. Our discussions led to the conclusion that the short-term variations of Jupiter's Decimetric brightness distribution may be the result of submicrometre charged dust particles undergoing significant electromagnetic perturbations while interacting with the radiation-belt electrons in the Jovian ring's innermost component.
\end{abstract}

Key words. radiation mechanisms: non-thermal - solar system: general - radio continuum: solar system

\section{Introduction}

In the late 1950s, measurements of Jovian emission in the Decimetric radio band provided evidences of relativistic electrons magnetically trapped close to the planet Jupiter (Radhakrishnan \& Roberts 1960; Morris \& Berge 1962). This population of energetic electrons was quickly identified as the "Van Allen" radiation belt for Jupiter. Shortly after its discovery, Jupiter's radiation-belt emission has been monitored in the cm wavelength band with single dish (Gerard 1970, 1976; Klein 1976; Klein et al. 1972, 1989, 2001; Miyoshi et al. 1999; Galopeau \& Gerard 2001; Tsuchiya et al. 2009) and its spatial distribution around the planet has been examined with aperture arrays (de Pater 1980; de Pater \& Jaffe 1984; Sault et al. 1997; Bolton et al. 2002; Kloosterman et al. 2008).

The first two-dimensional brightness distribution maps of the synchrotron-emitting region were provided by Berge (1966) and Branson (1968) and pointed out the presence of a confined electron population at the equator close to Jupiter. Later, higher resolution interferometric maps displayed the regions of intense emission along the equator and at higher latitudes (de Pater et al. 1982; de Pater \& Dickel 1986; Leblanc et al. 1997; Santos-Costa 2001; de Pater \& Dunn 2003). The features of such a brightness distribution have been recently interpreted by Santos-Costa \& Bolton (2008).

Data analysis of Jupiter's synchrotron radio emission demonstrated that the Jovian radiation zones exhibit important temporal variability on different time-scales. Measurements of the total flux density at $13-\mathrm{cm}$ wavelength over 40 years show fluctuations as large as 25 to $50 \%$ on time-scales of months to years (Carr et al. 1983; Klein et al. 2001), and detailed analyses of observations at other wavelengths demonstrate changes in intensity on scales of weeks to months (Roberts \& Huguenin 1962; Gerard 1970, 1976; Gulkis et al. 1973; Berge 1974; Klein 1976; Klein et al. 1972, 1989; de Pater \& Klein 1989; Bolton 1990). Until the collision of comet Shoemaker-Levy/9 with Jupiter in July 1994, short-term variations on time-scales of days to weeks were never measured with single dish or witnessed with aperture array (Bolton et al. 2004).

In this paper, we present the first evidence of short term variations (days to weeks) of Jupiter's radiation-belt emission obtained with interferometric measurements. Over a two-month period of observational time in 2002 (October to December), the Jovian synchrotron emission was observed with the Very Large Array (VLA). We first present the data processing and discuss our imaging results. Then, we present proof of how short-term 
Table 1. VLA 2002 observational time schedule and approximate CML coverage (Sect. 2).

\begin{tabular}{cccc}
\hline \hline Month & Day & Time (UT) & CML range $\left(^{\circ}\right)$ \\
\hline October & 28 th & $12: 00$ to $14: 30$ & {$[345 ; 75]$} \\
November & 1 st & $10: 00$ to $12: 10$ & {$[154 ; 232]$} \\
November & 5 th & $08: 00$ to $19: 30$ & All \\
November & 8th & $10: 00$ to $12: 00$ & {$[127 ; 200]$} \\
November & 10 th & $10: 45$ to $13: 00$ & {$[96 ; 177]$} \\
November & 11 th & $16: 40$ to $19: 00$ & {$[101 ; 185]$} \\
November & 21 th & $17: 20$ to $19: 20$ & {$[190 ; 263]$} \\
December & 4 th & $10: 00$ to $12: 30$ & {$[82 ; 173]$} \\
December & 11 th & $09: 45$ to $12: 30$ & {$[47 ; 147]$} \\
\hline
\end{tabular}

variability can manifest in brightness distribution variations as a function of Jovian System III longitude.

\section{Observations and data reduction analysis}

Between late October and early December 2002, Jupiter was observed at $6 \mathrm{~cm}(5 \mathrm{GHz})$ and $20 \mathrm{~cm}(1.5 \mathrm{GHz})$ with the VLA. During this campaign of observations, the antenna locations were the same with a maximum separation between antennas of 3.6 kilometers (C-configuration) (NRAO Green Book, 1992). The Earth's declination $\left(D_{\mathrm{E}}\right)$ as seen from Jupiter varied between 0.34 and 0.07 degree jovigraphic. Jupiter's distance from Earth was $\sim 5 \pm 0.3 \mathrm{AU}$ and its angular size was approximately $39.4^{\prime \prime}$. The observational times were spread out on 9 days as Table 1 shows. On 5 November 2002, the measurements lasted $11.5 \mathrm{~h}$. For the other eight days, Jupiter was observed during two to three hours. Given Jupiter's $9^{\mathrm{h}} 55^{\mathrm{m}}$ period, a full planet rotation could only be observed during the day of 5 November 2002. Figure 1 shows the central meridian longitudes (CMLs) monitored during the different observational days. Longitudes in the $[\sim 265 ; \sim 345]$ degree range values were observed once during the campaign of observations, and those in the $[\sim 230$; $\sim 265]$ and $[\sim 345 ; \sim 45]$ degree range values at two different days. Longitudes in the $[\sim 45 ; \sim 230]$ degree range values were monitored from three to six times. The coverage in CML for each observation day is summarized in Col. 4 of Table 1.

The all four Stokes parameters were measured during the whole campaign of radio observations. The UV data were flagged with NRAO AIPS (Astronomical Image Processing Software) and reduced with the MIRIAD (Multichannel Image Reconstruction Image Analysis and Display) package software (Sault et al. 1995). The radio sources $0842+324$ and $1331+305$ were used as calibrators (Perley \& Taylor 2003). Data at 6-cm wavelength were self-calibrated using a uniform limb-darkened model of the planet's disk (Sault et al. 1995). After being calibrated, the visibility data were step-by-step processed (using the MIRIAD tasks INVERT, MAXEN, and RESTOR) to produce maps of Jupiter's Decimetric radiation. During the imaging process, the thermal emission was subtracted. The brightness temperatures of Jupiter at $6 \mathrm{~cm}$ and $20 \mathrm{~cm}$ were respectively set to 240 and $350 \mathrm{~K}$ in the two-parameter disk model used for removing the planet's thermal emission (Sault et al. 1995). Multifrequency synthesis was used as well to incorporate the independent channels across the $50 \mathrm{MHz}$ bandwidth of the $\mathrm{C}$ - and L-band. The intensity of the brightness temperature was converted from Jansky (Jy) to Kelvin (K) units and normalized to a geocentric distance of $4.04 \mathrm{AU}$.

We concede a $5 \%$ error on the brightness temperatures of the "Clean" images, primarily resulting from the calibration and subtraction methods. The given error on the temperature
Table 2. VLA 1997 observational time schedule and approximate CML coverage (Sect. 3.1).

\begin{tabular}{cccc}
\hline \hline Month & Day & Time $($ UT) & CML range $\left({ }^{\circ}\right)$ \\
\hline May & 6th & $10: 00$ to $17: 30$ & {$[185 ; 97]$} \\
May & 7 th & $10: 00$ to $15: 15$ & {$[335 ; 275]$} \\
May & 11 th & $10: 15$ to $17: 00$ & {$[227 ; 112]$} \\
May & 12 th & $09: 45$ to $17: 00$ & {$[0 ; 262]$} \\
\hline
\end{tabular}

is an upper limit determined from the inverse of the signal to noise ratio. The noise is calculated with the root mean square method during the reduction of the visibility data sets with the MIRIAD task INVERT. The brightness temperature $\Delta T_{\mathrm{b}}$ corresponding to the rms noise $\sigma_{\mathrm{S}}$ is $\Delta T_{\mathrm{b}}=\left(\sigma_{\mathrm{S}} / \Omega_{\mathrm{A}}\right)\left(\lambda^{2} / 2 \mathrm{k}\right)$, with $\Omega_{\mathrm{A}}=\pi \theta_{\mathrm{o}}^{2} /\left(4 \log _{e}(2)\right)$ the beam solid angle of a Gaussian beam with half-power width $\theta_{\mathrm{o}}$ (NRAO Green Book, 1992). For the VLA 2002 campaign, $\Omega_{\mathrm{A}}$ is approximately $5 \times 10^{-5} \mathrm{Jy} /$ beam for both wavelengths and $\theta_{\mathrm{o}}$ is about $4^{\prime \prime}$ (respectively $13^{\prime \prime}$ ) for the wavelength of $6 \mathrm{~cm}$ (respectively $20 \mathrm{~cm}$ ). The temperature corresponding to the rms noise at 6 (resp. at 20) $\mathrm{cm}$ is about 0.15 (resp. 0.19) Kelvin. The emission from Jupiter's radiation belts is detected above the sky background which has a brightness temperature of 3 to $4 \mathrm{~K}$ in the decimetric band, and can reach temperatures approaching hundreds of Kelvin. The 5\% error on the brightness temperatures of the "Clean" maps can be thus justified: $(0.15$ to $0.19 \mathrm{~K}) /(3$ to $4 \mathrm{~K}) \approx 4$ to $6 \%$.

\section{Data processing results}

\subsection{Rotation-averaged images}

Figure 2 shows four longitude-averaged images of the total intensity. The right-hand panels are the maps of Jupiter's radiationbelt emission at $20 \mathrm{~cm}$ (top panel) and $6 \mathrm{~cm}$ (bottom panel) constructed from the measurements made in 2002. Panels on the left-hand side of Fig. 2 display the spatial distribution of the same radio source at the same wavelengths, but monitored with the VLA five years earlier. In May 1997, the VLA was in the Bconfiguration. $D_{\mathrm{E}}$ was close to $0^{\circ}$ and Jupiter was approximately 5 AU away from the Earth (Santos-Costa 2001). Table 2 provides further information regarding the VLA 1997 campaign.

Longitude-averaged images are constructed essentially by assuming that the radiation region is circularly symmetric about Jupiter's tilted magnetic axis, and no large temporal change in the radiation belts emission is happening during the observations. The method for constructing longitude-averaged maps is described in the work of Leblanc et al. (1997). In Fig. 2, the images have the magnetic axis vertical. Each map was built by combining the measurements obtained for each wavelength during a VLA campaign of observations. The effects of the magnetic field wobble were corrected (see Leblanc et al. 1997).

In May 1997, the VLA B-configuration provided a good spatial resolution of the radiation around Jupiter. The synthesized beam for the longitude-averaged images was $\sim 3.1^{\prime \prime} \times 3.1^{\prime \prime}$ at $6 \mathrm{~cm}$ and $5.2^{\prime \prime} \times 3.1^{\prime \prime}$ at $20 \mathrm{~cm}$. From the measurements made in 1997, the constructed high-resolution maps at 6 and $20 \mathrm{~cm}$ clearly show the regions of intense emission along the magnetic equator with a peak located at L-shell $\sim 1.4 R_{\mathrm{J}}$, and the secondary radiation peaks located at higher latitudes. The C-configuration of the VLA during Fall 2002 could not provide an image resolution as good as the B-configuration did in 1997. For the 2002 campaign of observations, the synthesized beam was $~ 3.8^{\prime \prime} \times 3.6^{\prime \prime}$ at $6 \mathrm{~cm}$ and $12.6^{\prime \prime} \times 12.1^{\prime \prime}$ at $20 \mathrm{~cm}$. Only maps at $6-\mathrm{cm}$ wavelength have a resolution high enough to provide 


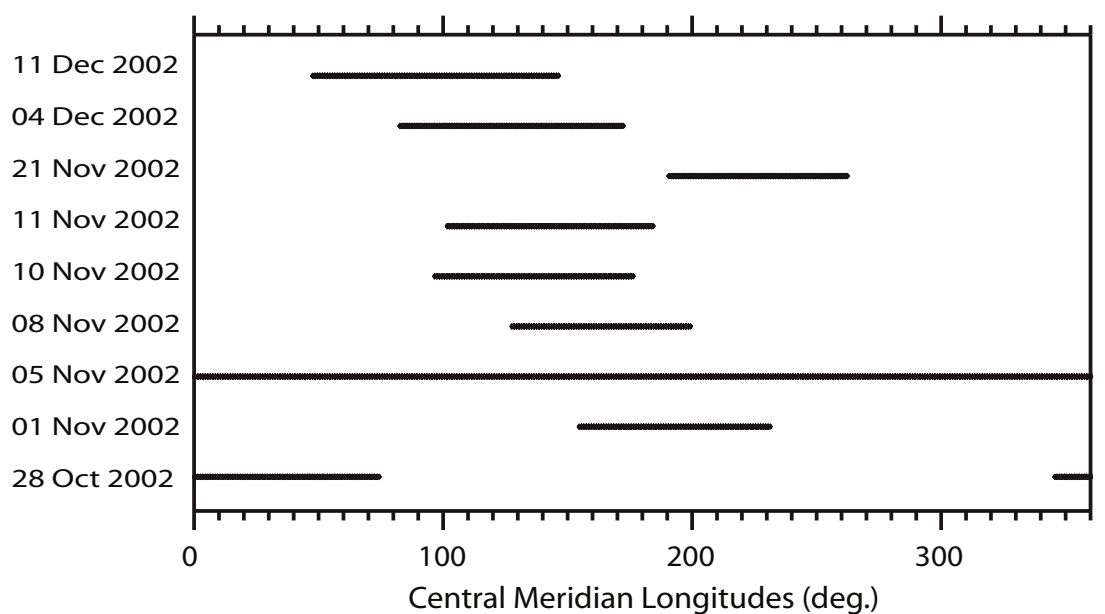

Fig. 1. Central meridian longitudes coverage for each observation day of the VLA 2002 campaign (Sect. 2).
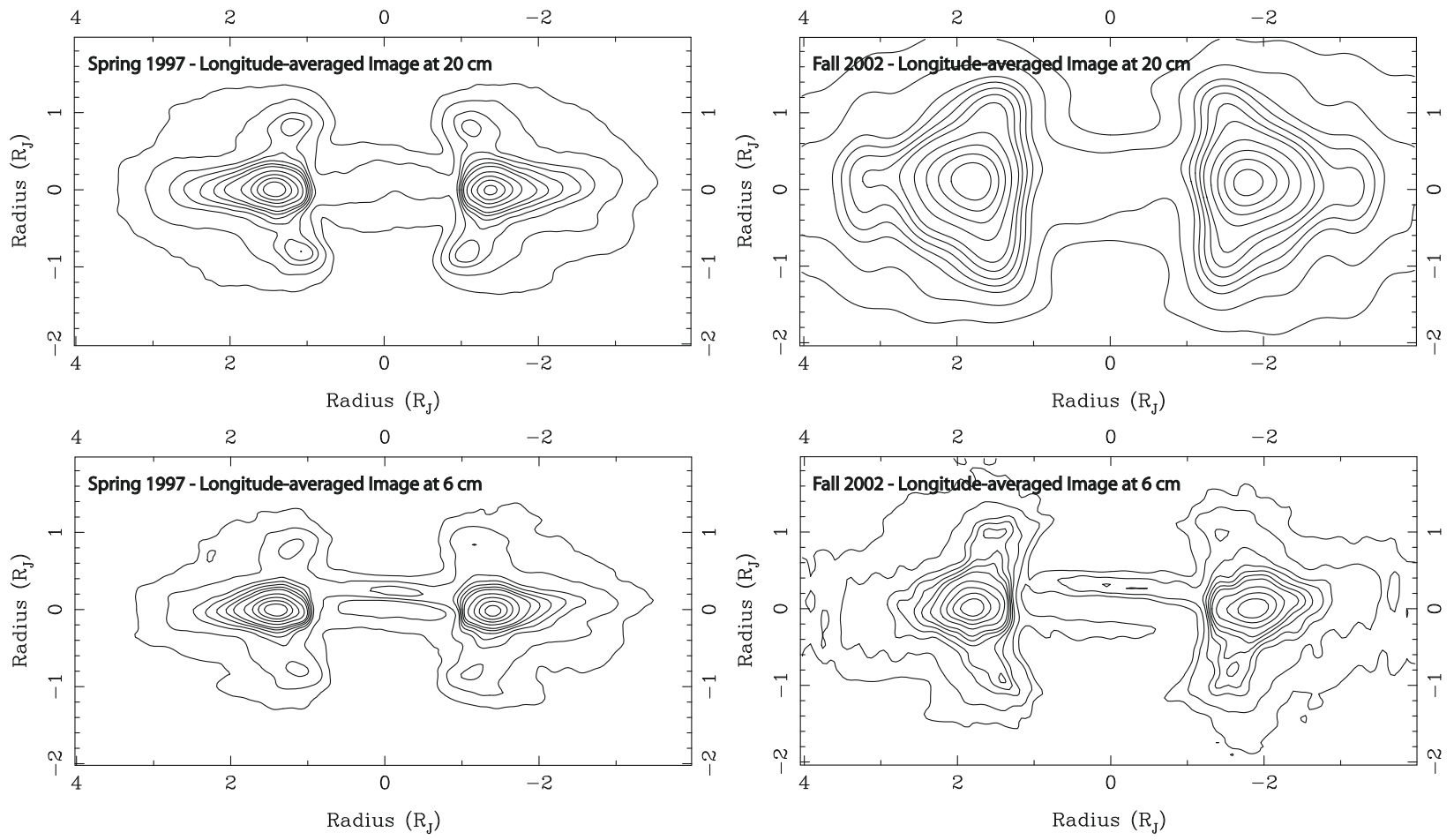

Fig. 2. Rotation-averaged images of Jupiter's radiation-belt emission at $20 \mathrm{~cm}$ (top panels) and $6 \mathrm{~cm}$ (bottom panels) in total intensity. The two-dimensional maps on the right-hand (respectively left-hand) side were constructed from the measurements made with the VLA in 2002 (respectively in 1997). For each map, contours correspond to 10, 20, 30, 35, 40, 45, 50, 60, 80, and 90\% of the radiation peak (see Sect. 3.1).

detailed brightness distributions of Jupiter's radiation-belt emission. For all the images of Fig. 2, the peak brightness distribution is near the equator and on the left-hand side of the planet.

\subsection{Images at different longitudes}

For each of the 9 days of observation in 2002, we have formed two-dimensional maps of the total intensity of Jupiter's synchrotron radio emission. Images were constructed every $10^{\circ}$ in longitude. For each longitude, data within $\pm 10^{\circ}$ of the CML were used. Due to the limited time of observations for 8 of the 9 days in 2002, only series of six to eight images at 6- and $20-\mathrm{cm}$ wavelength were produced. For the day of 5 November 2002, thirty four brightness distributions were reconstructed for both wavelengths from the measurements. Again, the effects of the magnetic field wobble were corrected (see Leblanc et al. 1997).
Images for the VLA 1997 campaign were constructed using the same approach (Santos-Costa 2001). To allow comparisons between images from different periods of time, the VLA 1997 campaign images were degraded to the image resolution of the campaign of observations made in 2002. Making the image resolutions match was performed using the MIRIAD task CONVOL (Sault et al. 1995).

Figure 3 maps the brightness distribution for the CML of $70^{\circ}$ and the wavelength of $6 \mathrm{~cm}$. The bottom panels are the spatial distributions reconstructed from the VLA 2002 campaign for two different days. Compared to the day of 5 November 2002 (bottom left-hand panel), the equatorial brightness distribution of the east lobe (on the left-hand side of the planet) is brighter than the west lobe (on the right-hand side of the planet) during the day of 11 December 2002 (bottom right-hand panel). Based on the past observations (Santos-Costa 2001) displayed 

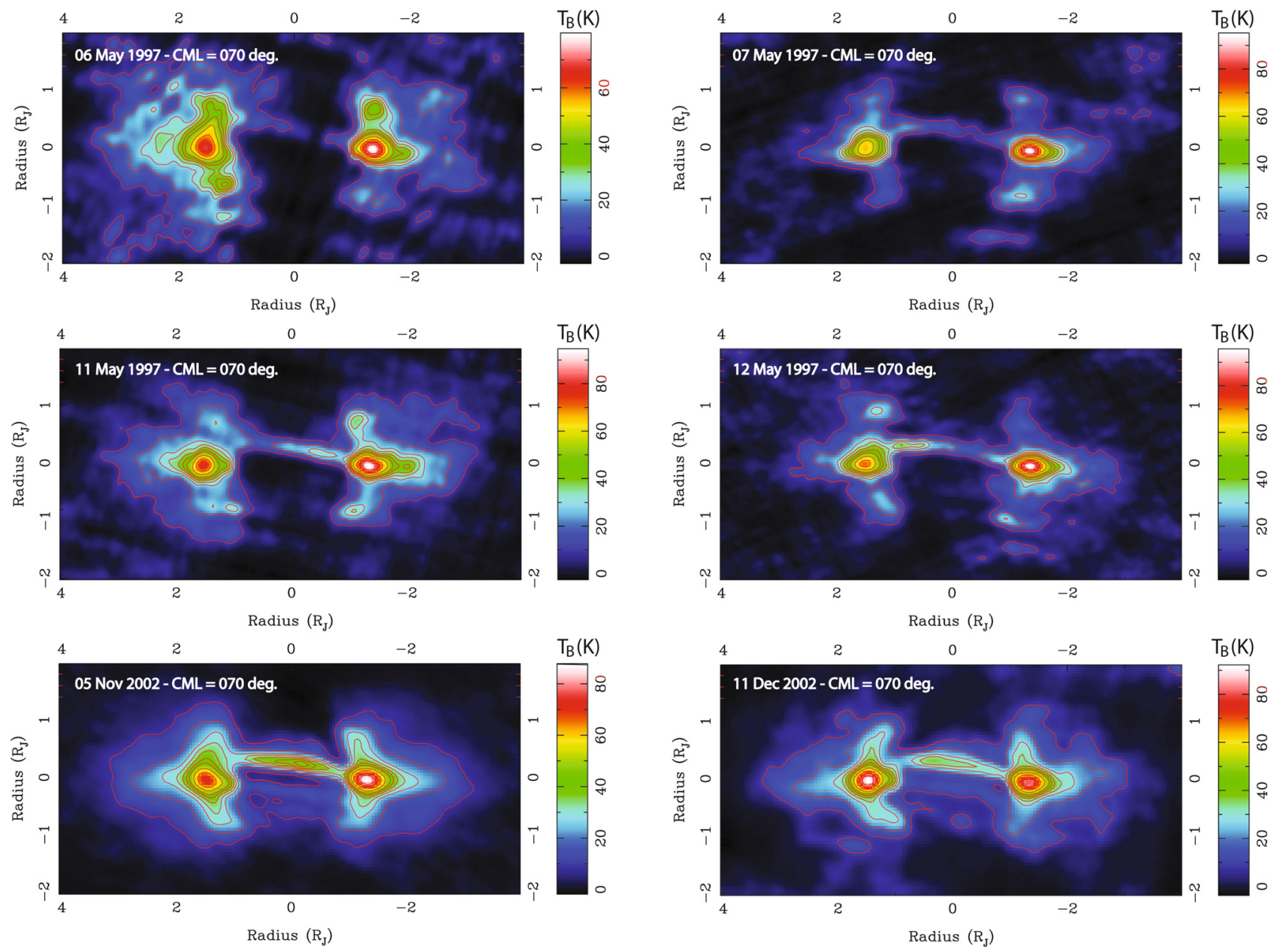

Fig. 3. Two-dimensional images of Jupiter's radiation-belt emission at 6-cm wavelength $\left(\mathrm{CML}=70^{\circ}\right.$ ). On 11 December 2002 (bottom right-hand panel), the emissivity from the east lobe became temporally brighter than the west lobe, resulting in a shift of the peak brightness distribution of the emission to the other side of the planet (significantly different from what was observed 3-weeks earlier on 5 November 2002 (bottom left-hand panel) and in May 1997 (upper four panels)). See Sect. 3.2.

on the top four panels of Fig. 3, the two-dimensional images at $70^{\circ} \mathrm{CML}$ are more like the interferometric map which displays the brighter lobe located on the right-hand side of the planet on 5 November 2002. Figure 3 thus demonstrates the first evidence of short-term variability in Jupiter's radiation-belt emission on a time-scale of weeks with VLA observations. For the CML of $70^{\circ}$, the peak brightness distribution of Jupiter's synchrotron emission at $6-\mathrm{cm}$ wavelength temporally shifted from one side of the planet to the other side due to a significant enhancement of the emission peak on the east side of the planet.

Figure 4 provides further proof for short-term changes in Jupiter's radiation-belt emission at the wavelength of $6 \mathrm{~cm}$. The top left panel is the brightness emission for the day of 12 May 1997 and $\mathrm{CML}=120^{\circ}$. The other five maps display the synchrotron emission as distributed around the planet at the same longitude and wavelength, but for different days of Fall 2002. In May 1997, the peak brightness distribution at the CML of $120^{\circ}$ was located on the east side of the planet. In November and December 2002, the emission peak was observed with the VLA to shift back and forth from one side of the planet to the other. During the first weeks of November 2002, Jupiter's synchrotron radiation gradually became brighter on the west side of the planet (west lobe). The emission peak shifted to the other side due to a clear decrease of the intensity of the radiation on the east lobe. Between mid-November and mid-December, the emissivity of the east lobe became brighter and brighter along with the weeks. On 11 December 2002, the peak brightness distribution was observed to be on the east side of the planet, as it was the case on 12 May 1997.

Figure 5 shows another example of temporal variations in Jupiter's synchrotron radio emission on time-scales of days to weeks. As with Fig. 4, we compared the brightness distribution of the radiation-belt zone at $6-\mathrm{cm}$ wavelength between the day of 12 May 1997 and few days in November and December 2002. In Fig. 5, the CML of $130^{\circ}$ is the longitude now examined. On 12 May 1997, the peak brightness distribution at $130^{\circ} \mathrm{CML}$ was on the west (right-hand) side of the planet. On 5 November 2002, the radiation peak was still located on the right-hand side of Jupiter. However, the equatorial brightness on the east (lefthand) side started radiating a brighter emission which became almost comparable to the emissivity radiated from the west side. On 10 November 2002, the east lobe was as bright as the west lobe. The following day, the emission peak was again observed on the west (right-hand) side of Jupiter. Approximately three weeks later (on 4 December 2002), the radiation peak shifted to the east side. Finally, on 11 December 2002, the peak brightness emission was still located on the east side of Jupiter. Unlike Fig. 4 and the CML of $120^{\circ}$, the emissivity peak at $130^{\circ}$ on 11 December 2002 was not radiated from the west lobe as it was the case on 12 May 1997. 

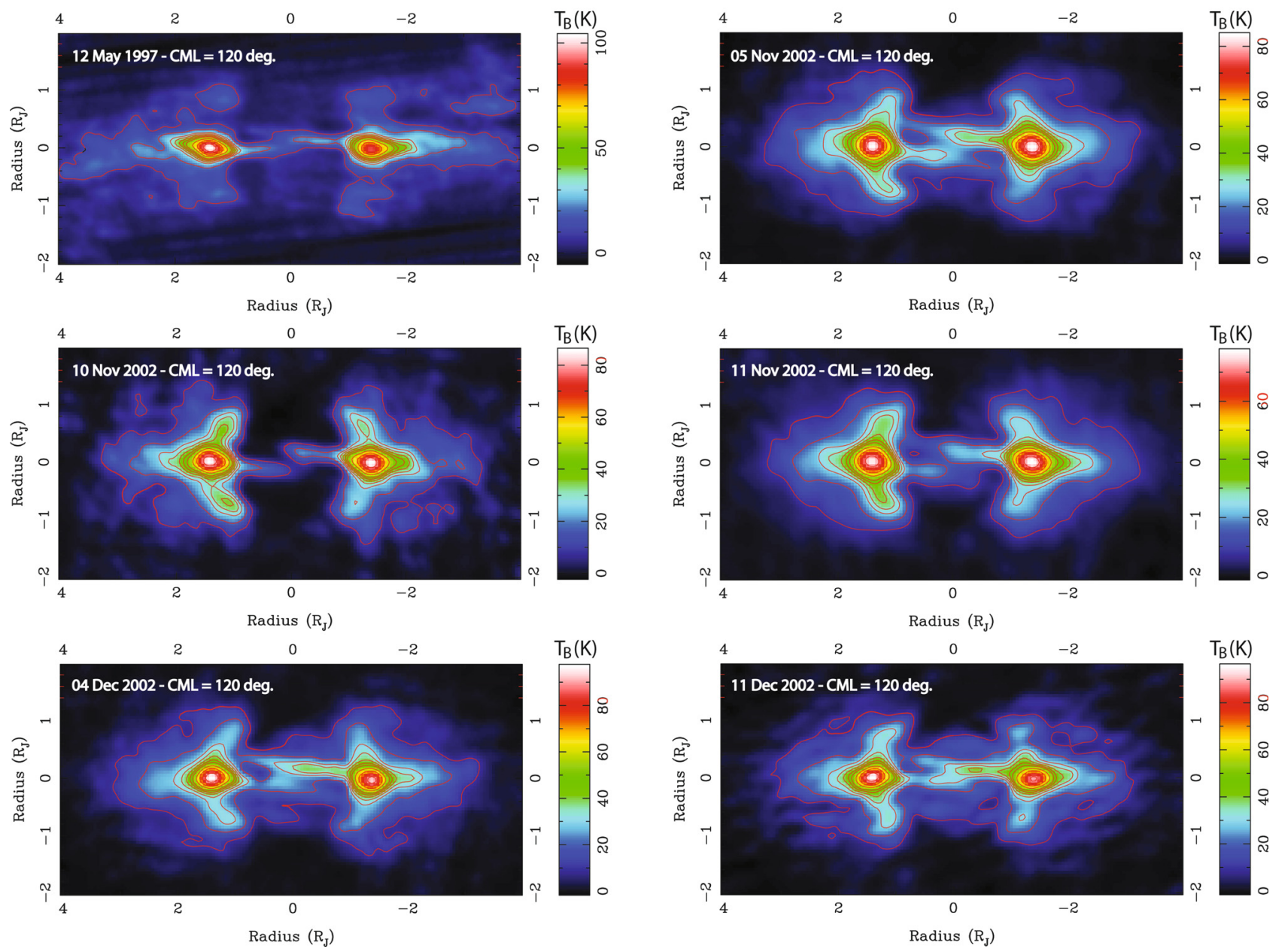

Fig. 4. Two-dimensional images of Jupiter's radiation-belt emission at $6-\mathrm{cm}$ wavelength $\left(\mathrm{CML}=120^{\circ}\right)$. In May 1997 , the peak brightness distribution was located on the left-hand side of the planet. In November and December 2002, the emission peak was observed with the VLA to shift back and forth from one side of the planet to the other (see Sect. 3.2).

\subsection{Changes in the peak brightness distribution}

From the maps constructed at 6-cm wavelength, the temporal changes in the brightness temperature $T_{\mathrm{B}}$ as measured along the magnetic equator are examined. Figure 6 displays the onedimensional brightness scans we have obtained for the CML of $20^{\circ}, 90^{\circ}, 170^{\circ}, 190^{\circ}, 210^{\circ}$ and $320^{\circ}$ when taking a slice through the Jovian magnetic equator. Figure 7 shows the dependence of $T_{\mathrm{B}}$ as a function of the position along the magnetic equator for the CML of $70^{\circ}, 120^{\circ}, 250^{\circ}, 270^{\circ}, 300^{\circ}$ and $340^{\circ}$. For each panel (or CML) of Figs. 6 and 7, the brightness temperatures between different days of observation are compared. Table 3 provides the color legend for Figs. 6 and 7 and specifies the observational days as well.

Figure 6 actually shows one-dimensional scans of the equatorial brightness at $6-\mathrm{cm}$ wavelength for days of observation when a shift of the peak brightness distribution from one side of the planet to the other side was neither observed nor confirmed. Although the intensity of the emission peaks on each side of the planet were fluctuating from one observational day to another one by five to thirty points of percentage, the brightness peak stayed located on the same side of the planet.

The top left-hand panel of Fig. 7 validates the observation of changes in the peak brightness distribution at the wavelength of $6 \mathrm{~cm}$ and CML of $70^{\circ}$ (Fig. 3). The brightness peak was located on the right-hand side of the planet in October and November
Table 3. Color Legend for Figs. 6 and 7 (Sect. 3.3).

\begin{tabular}{|c|c|c|c|}
\hline Observation Time & / Curve Color & Observation Time & Curve Color \\
\hline 06 May 1997 & 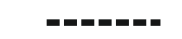 & 08 Nov 2002 & \\
\hline 07 May 1997 & -Ex=E- & 10 Nov 2002 & \\
\hline 11 May 1997 & 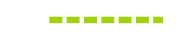 & 11 Nov 2002 & |-10010010 \\
\hline 12 May 1997 & 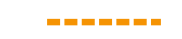 & 21 Nov 2002 & 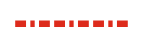 \\
\hline 28 Oct 2002 & & 04 Dec 2002 & 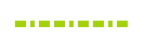 \\
\hline 01 Nov 2002 & & $11 \mathrm{Dec} 2002$ & \\
\hline 05 Nov 2002 & & & \\
\hline
\end{tabular}

2002, as it was in May 1997. Between the two campaign of observations, the emission peaks on both sides of Jupiter increased between 10 and 25\%. In December 2002, the brightness peak shifted from the right-hand side of the planet to the left-hand side due to an enhancement of the east lobe's emission peak of $\sim 30 \%$ and a decrease of the west lobe's emission peak of $\sim 15 \%$.

The top right-hand panel of Fig. 7 confirms the fluctuations of the brightness distribution that were observed for the CML of $120^{\circ}$ (Fig. 4). During the two campaigns of observations with the VLA, the brightness peak was observed on the left-hand side of Jupiter except for the 5th, 10th and 11th of November 2002. The brightness peak temporally shifted to the other side in November 

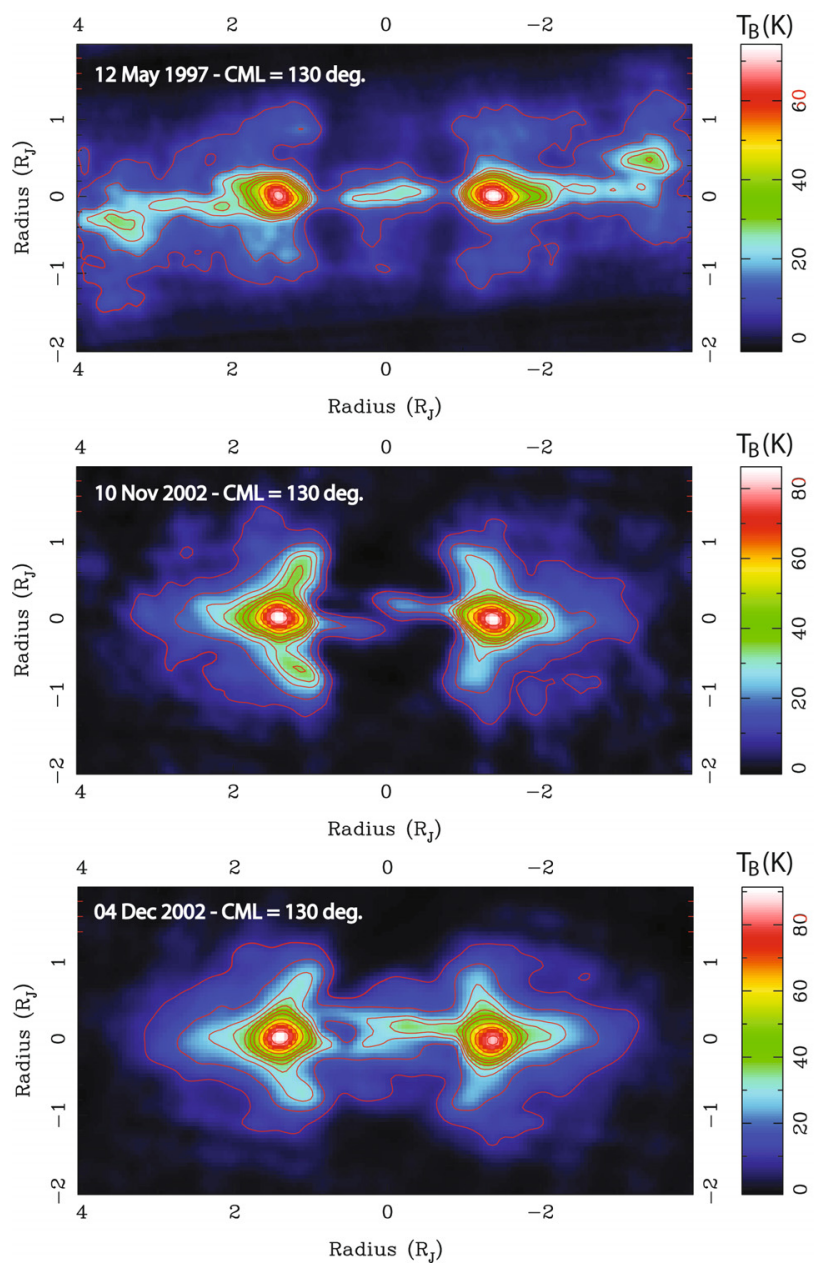
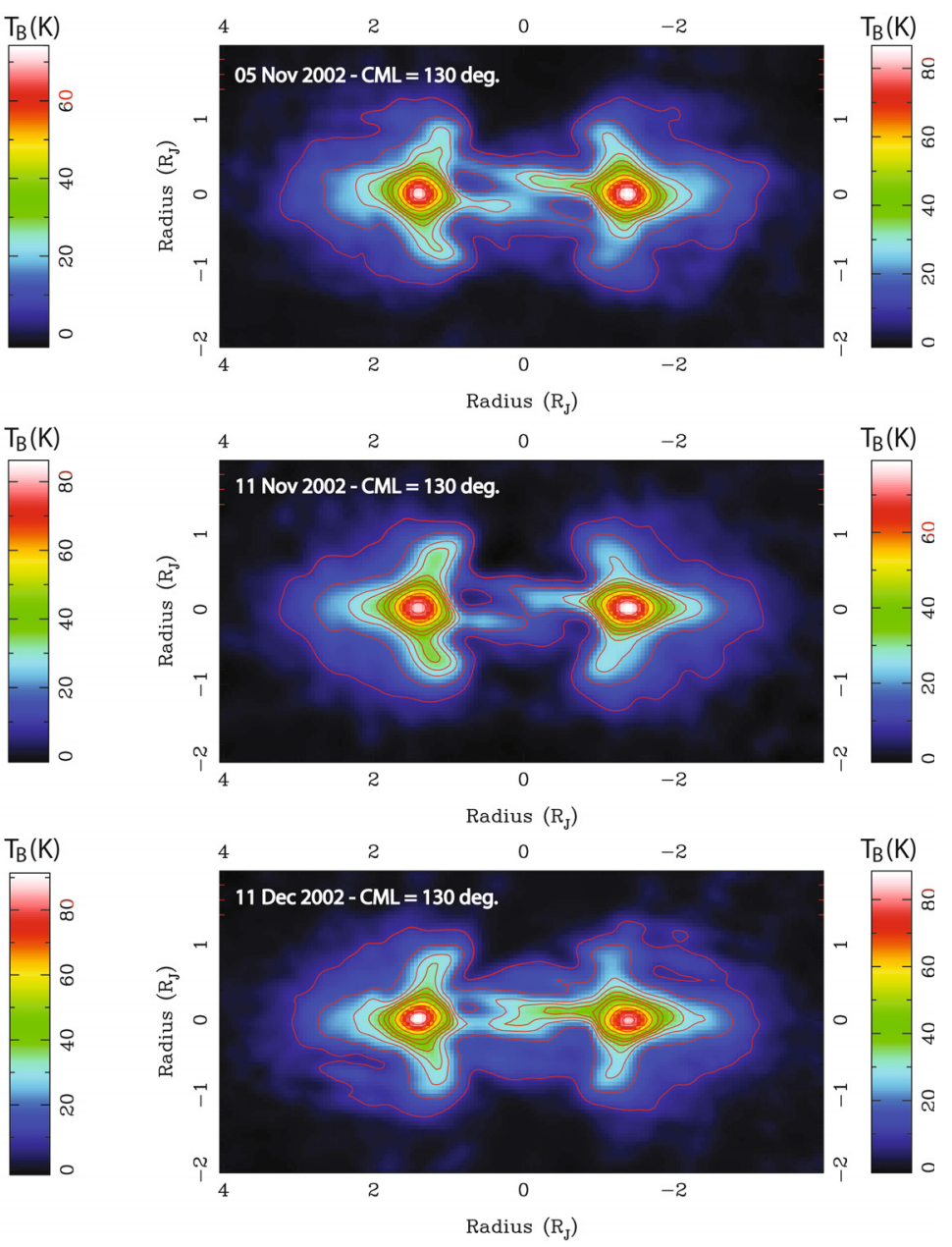

Fig. 5. Two-dimensional images of Jupiter's radiation-belt emission at 6-cm wavelength $\left(\mathrm{CML}=130^{\circ}\right)$. On 12 May 1997 and 5 November 2002 , the radiation peak was located on the right-hand side of Jupiter. On 10 November 2002, the east lobe was as bright as the west lobe. Approximately three weeks later (on 4 December 2002), the radiation peak shifted to the left-hand side of the planet and was still located on this side of Jupiter on 11 December 2002 (see Sect. 3.2).

2002 due to a decrease in the intensity of the east lobe's emission peak of $\sim 20 \%$.

The middle left-hand panel of Fig. 7 is an example of changes in Jupiter's Decimetric radiation only observed during the VLA 1997 campaign. For the CML of $250^{\circ}$, the switch of the brightness peak from one side of the planet to the other occurred between the day of 06 May 1997 and day of 12 May 2002. The emission peak on the right-hand side (west lobe) first increased by $\sim 26 \%$ in five days; then it decreased by $10 \%$ within one day. Regarding the emission peak on the other side of the planet (east lobe), we first observed a decrease in the intensity of radiation then an enhancement. The sudden rise of the peak brightness distribution on the right-hand side was enough to make the west lobe become temporally brighter.

The one-dimensional scans of the equatorial brightness for the CMLs of $270^{\circ}$ and $300^{\circ}$ show variability of Jupiter's synchrotron radiation in November 2002. For the CML of $270^{\circ}$ (middle right-hand panel of Fig. 7), the equatorial brightness distribution on the left-hand side of the planet (east lobe) became brighter in 2002 when the emission peak of the west lobe decreased in intensity by more than $40 \%$ ( $T_{\mathrm{B}}$ dropping from $\sim 108$ to $\sim 76 \mathrm{~K}$ ) while the emission peak of the east lobe only decreased by $15 \%$ ( $T_{\mathrm{B}}$ dropping from $\sim 92$ to $\left.\sim 80 \mathrm{~K}\right)$. For the CML of $300^{\circ}$ (lower left-hand panel of Fig. 7), the emission peak of the east lobe barely fluctuated between the two VLA campaigns of observations. The shift of the brightness peak from the left to the right-hand side of Jupiter was the result of an increase of the emission peak's intensity of $\sim 14 \%$. For the CML of $340^{\circ}$ (lower right-hand panel of Fig. 7) changes in the brightness distribution likely occurred in May 1997 and November 2002.

\section{Angular sectors for source of variability}

Radiation from the lobes are generated by near-equatorial highenergy electrons magnetically trapped between Jupiter's surface and the orbit of Amalthea $\left(\sim 2.5\right.$ planetary radius $\left.\left(R_{\mathrm{J}}\right)\right)$ (Santos-Costa \& Bolton 2008). Due to the latitudinal confinement of this electron population in a region of strong magnetic field strength, brightness temperatures along the magnetic equator are greater than $30 \%$ of the brightness peak for radial distances ranging from 1 to $2 R_{\mathrm{J}}$. The emission peak is typically radiated near $1.4 R_{\mathrm{J}}$. Because the strength of the planetary magnetic field $B$ along the equator is $1 / r^{3}$ dependent (dipolar approximation) and the synchrotron radiation is proportional to $\left(B \times\right.$ Energy $^{2}$, electrons populating L-shells in the $[1.35 ; 1.45]$ $R_{\mathrm{J}}$ range are likely the particles responsible for the emission peaks (with an intensity greater than $90 \%$ of the maximum) observed on both sides of the planet.

When the intensity of the peak brightness distribution of the east and west lobes at a given CML were examined in previous 

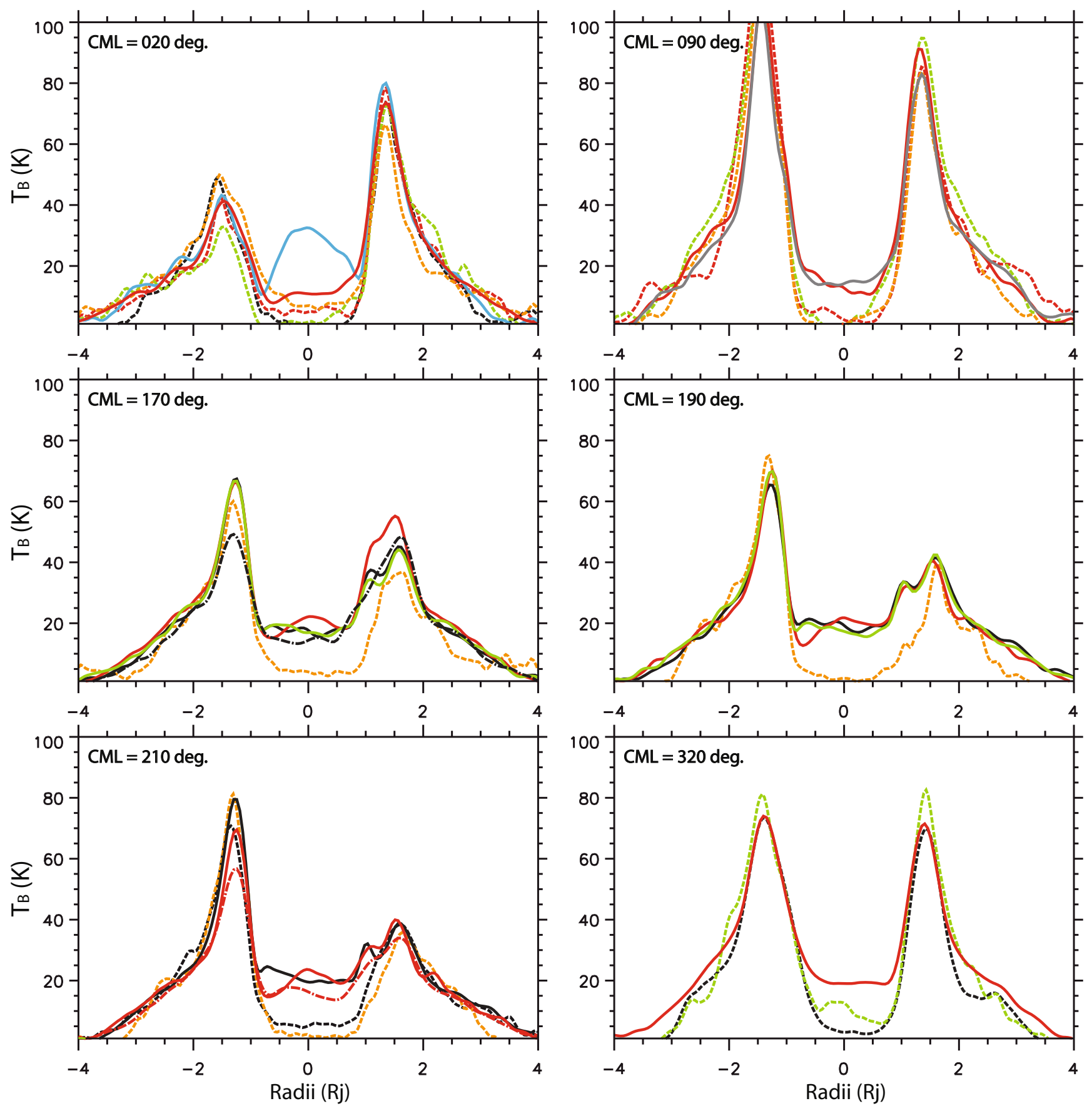

Fig. 6. Brightness temperature along the magnetic equator of the total intensity at $6 \mathrm{~cm}$ of Jupiter's radiation-belt emission for days of observation when a shift of the brightness peak from one side of the planet to the other side was neither observed (top four and lower left-hand panels) nor could be confirmed (lower right-hand panel) for a given CML (see Sect. 3.3).

works, switching from the longitude system CML to another one has proven to be helpful in understanding better the observations of Jupiter's synchrotron emission (see Leblanc et al. 1997; Dulk et al. 1997). The Jovian coordinate convention defines CML as the longitude of the central meridian with respect to the observer, while the longitude $\lambda_{\text {III }}$ is based on the "System III" prime meridian rotation angle of the magnetic field (Dessler 1983). By definition, Jupiter SIII Longitude $\lambda_{\text {III }}$ is independent of the observer. At a given CML, the emission peak on the right and left-hand sides of Jupiter are radiated by electrons drifting in the angular section $\Delta \lambda_{\mathrm{III}}=\mathrm{CML}-\left(90^{\circ} \pm \Delta \varphi\right)$ and $\mathrm{CML}+\left(90^{\circ} \pm \Delta \varphi\right)$, respectively. $\Delta \varphi$ is given by $\arccos (1.35 / 1.45)=20^{\circ}$. Table 4 summarizes the observation of changes in Jupiter's radiationbelt emission at $6 \mathrm{~cm}$ for the CMLs of $70^{\circ}, 120^{\circ}, 250^{\circ}, 270^{\circ}$, $300^{\circ}$ and $340^{\circ}$. Table 4 further indicates the $\lambda_{\text {III }}$ angular sectors where the fluctuations were generated.

Figure 8 traces the variations of the east-west peak brightness emission ratio for the two campaigns of observations as a function of CML. It is clearly shown that the variability of the lobes emissivity was actually observed for all CMLs. However, the shift of the brightness peak from one side of the planet to the other was observed at CMLs in the range from $70^{\circ}$ to $170^{\circ}$ (range R1) when the significant changes were located on the east lobe, and the range from $250^{\circ}$ to $350^{\circ}$ (range $\mathrm{R} 2$ ) for 

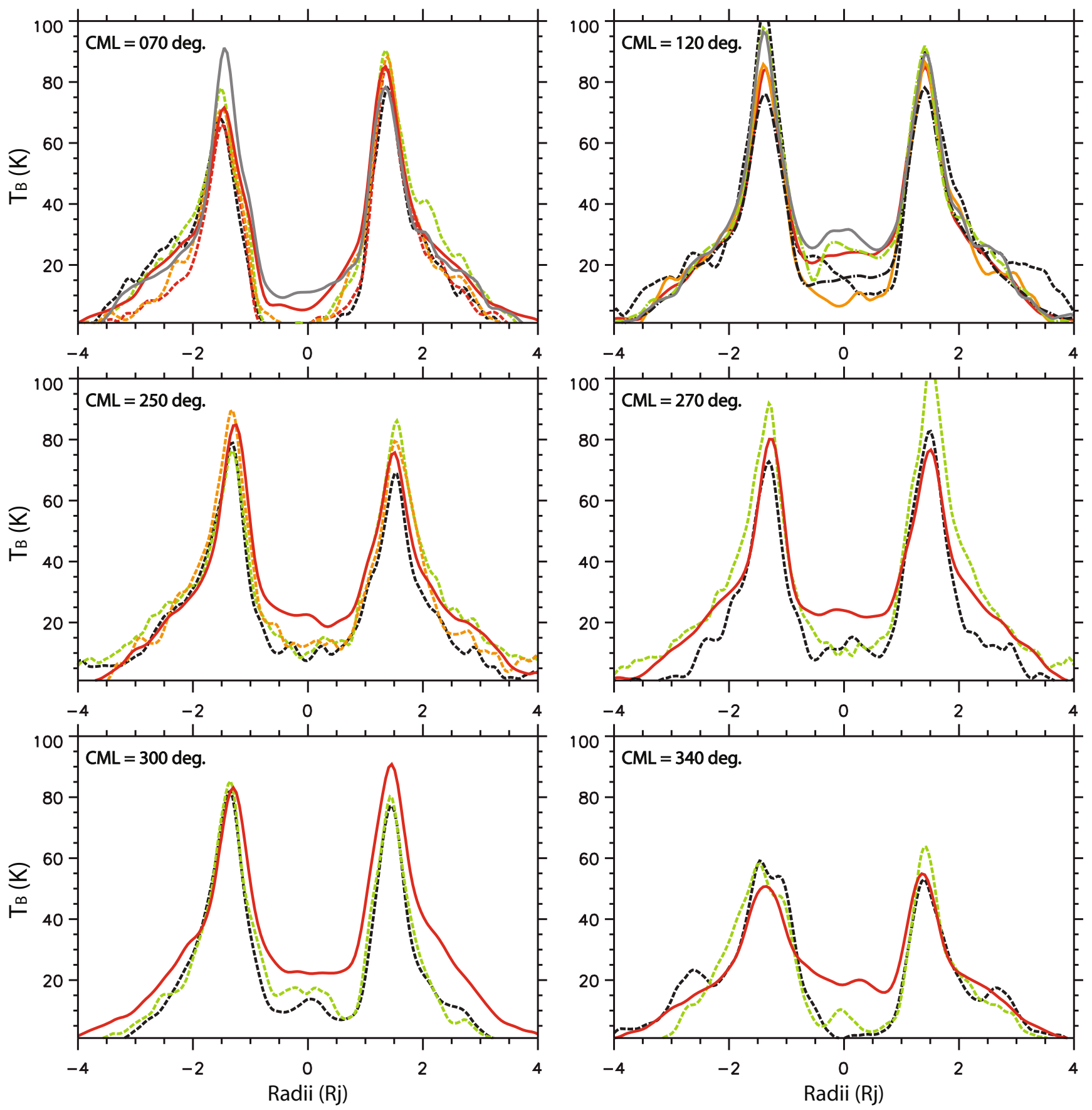

Fig. 7. Brightness temperature along the magnetic equator of the total intensity at $6 \mathrm{~cm}$ of Jupiter's radiation-belt emission for days of observation when a shift of the brightness peak from one side of the planet to the other side was observed for a given CML (see Sect. 3.3).

the other lobe. Given that the two-dimensional map for a given CML is constructed using data within $\pm 10^{\circ}$ of this longitude, the ranges $\mathrm{R} 1$ and $\mathrm{R} 2$ should be corrected by $+10^{\circ}$ (respectively $10^{\circ}$ ) for the left-hand (respectively right-hand) boundaries. Now, taking into account that the brightness distribution near the emission peaks is radiated by electrons drifting within angular sectors of $40^{\circ}, \mathrm{R} 1$ and $\mathrm{R} 2$ likely cover the longitudes in the ranges $[60 ; 180]$ and $[240 ; 360]$, respectively. These two ranges of CMLs actually correspond to the same range of $\lambda_{\mathrm{III}}$ longitudes $\left(\left[\lambda_{\mathrm{III}}^{1}\right]=[150 ; 270]\right.$ degree range $)$. The short-term changes in the peak brightness distribution on one side of the planet were even more significant when fluctuations on the other side were simultaneously occurring, meaning that the $\lambda_{\text {III }}$ longitude range $[60-90 ; 180-90] \equiv[240+90 ; 360+90] \equiv\left[330^{\circ} ; 90^{\circ}\right]=\left[\lambda_{\mathrm{III}}^{2}\right]$ is an angular sector contributing to the observation of changes in Jupiter's radiation-belt emission.

Inside $6 R_{\mathrm{J}}$, Jupiter's magnetic field is usually approximated to a dipole field with a magnetic moment of $B_{\mathrm{o}} \approx 4.28$ Gauss and tilted $\sim 9.4^{\circ}$ to the rotation axis toward the longitude $\lambda_{\text {III }}$ of $200^{\circ}$ in the northern hemisphere (Khurana et al. 2004). Figure 9 maps the total field intensity as predicted by the $O_{6}$ model at a radial distance of $1.4 R_{\mathrm{J}}$ (Connerney 1993). Because Jupiter possesses substantial quadrupole and octopole moments, the northsouth asymmetry of the field strength and its non axi-symmetry with longitude $\lambda_{\text {III }}$ are clearly observed at a jovigraphic distance of $1.4 R_{\mathrm{J}}$. The magnetic equator is a surface which sweeps the planetary latitudes in the $[-15 ;+10]$ degree range at $1.4 R_{\mathrm{J}}$. The red line on Fig. 9 delineates the equatorial surface where 


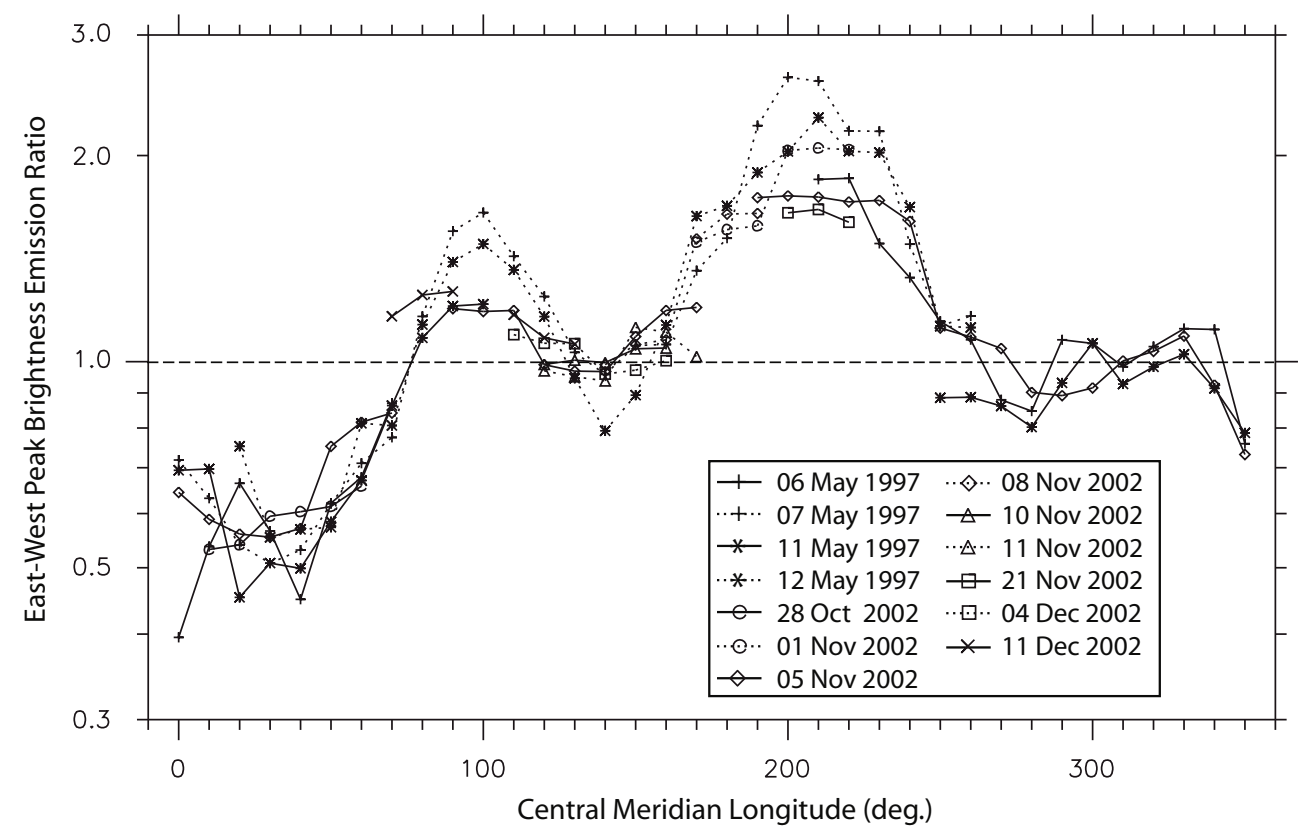

Fig. 8. Variations of the east-west peak brightness emission ratio observed during the VLA 1997 and VLA 2002 campaigns as a function of CML (see Sect. 4).

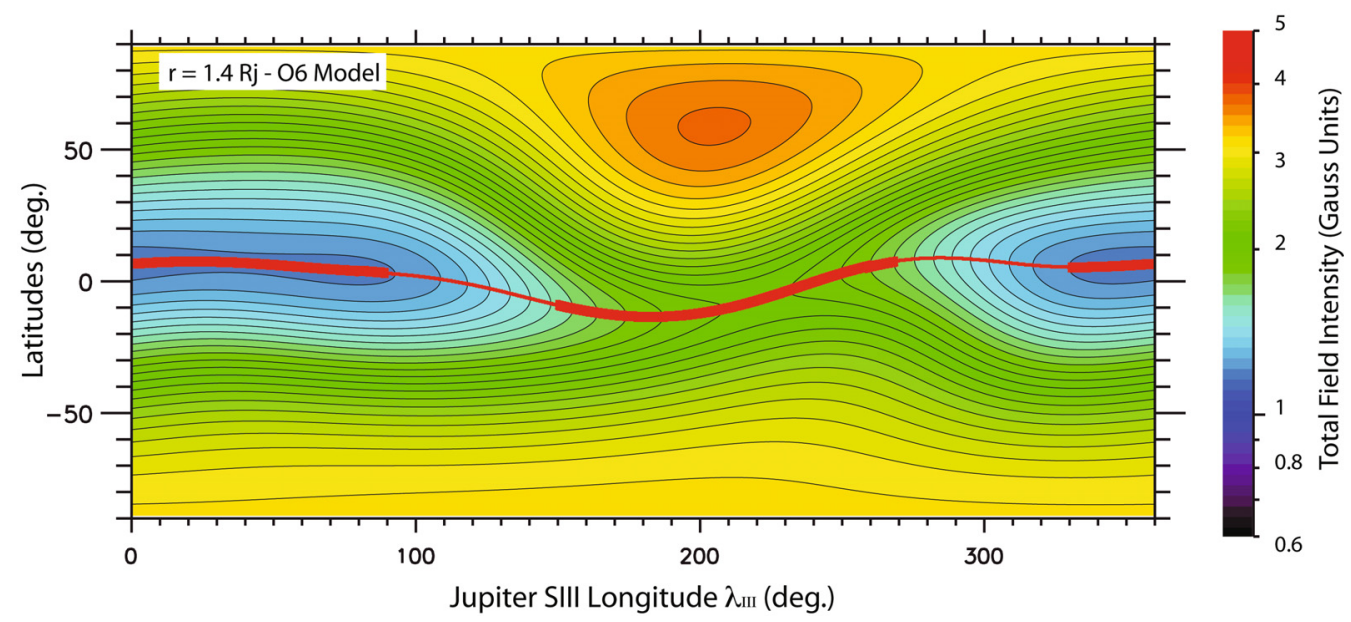

Fig. 9. Iso-contour map of the total field intensity as predicted by the $\mathrm{O}_{6}$ model at $1.4 R_{\mathrm{J}}$ (Connerney 1993). The red line follows the equatorial magnetic surface where the field strength is minimum. The angular sectors where the red line is thicker correspond to the longitude ranges where variations in Jupiter's radiation-belt emission peak were clearly observed (see Sect. 4).

the field strength is minimum. The thicker portions of this red line correspond to the angular sectors $\left[\lambda_{\mathrm{III}}^{1}\right]$ and $\left[\lambda_{\mathrm{III}}^{2}\right]$. One can point out that $\left[\lambda_{\mathrm{III}}^{1}\right]$ and $\left[\lambda_{\mathrm{III}}^{2}\right]$ cover the longitudes where the field strength along the equatorial surface is maximum and minimum, respectively.

\section{Concluding discussion and prospective}

In this paper, measurements of Jupiter Decimetric emission made in 2002 with the Very Large Array over a two-month period of observational time have been examined. From the observations made at the wavelengths of 6 and $20 \mathrm{~cm}$, we have constructed brightness distribution maps of the emission radiated around the planet by Jovian energetic electrons. Due to the C-configuration of the VLA during Fall 2002, the image resolution at $6 \mathrm{~cm}$ was $\sim 3.8^{\prime \prime} \times 3.6^{\prime \prime}$ compared to $12.6^{\prime \prime} \times 12.1^{\prime \prime}$ at $20 \mathrm{~cm}$, which made easier the analysis of the interferometric maps for the shorter wavelength.
The VLA images demonstrate significant changes in the spatial structure of Jupiter's radiation-belt emission on time-scales of days to weeks. The peak brightness distribution near the magnetic equator evolved differently during the 10-h rotation of the planet and over the campaign of observations. The comparisons of the two-dimensional maps at $6 \mathrm{~cm}$ with another campaign of observations made in May 1997 have confirmed our discovery of short-term variability of Jupiter's synchrotron emission with the VLA.

A detailed analysis of one-dimensional scans of the equatorial brightness has showed that, for a series of CMLs, the radiation peak was shifting back and forth from one side of the planet to the other on a time-scale of days. The change in the location of the emission peak was the result of fluctuations in the peak brightness distribution by $10 \%$ up to $40 \%$. Dulk et al. (1999) examined in detail how changes in the geometric parameter $D_{\mathrm{E}}$, the Earth's declination seen from Jupiter, can affect the 2-D brightness distribution and the longitude profile of the magnetic 
Table 4. Variability and location of the emission peaks at 6-cm wavelength (Sect. 4).

\begin{tabular}{clrlr}
\hline \hline & \multicolumn{2}{c}{ EAST LOBE } & \multicolumn{2}{c}{ WEST LOBE } \\
CML $\left(^{\circ}\right)$ & Emission peak & $\lambda_{\text {III }}\left({ }^{\circ}\right)$ & Emission Peak & $\lambda_{\text {III }}\left(^{\circ}\right)$ \\
\hline 070 & Increase $^{*}$ & $160 \pm \Delta \varphi$ & Decrease & $340 \pm \Delta \varphi$ \\
120 & Decrease & $210 \pm \Delta \varphi$ & Increase & $030 \pm \Delta \varphi$ \\
250 & Decrease & $340 \pm \Delta \varphi$ & Increase* & $160 \pm \Delta \varphi$ \\
270 & Increase & $000 \pm \Delta \varphi$ & Decrease* $^{*}$ & $180 \pm \Delta \varphi$ \\
300 & Stable & $030 \pm \Delta \varphi$ & Increase $^{*}$ & $210 \pm \Delta \varphi$ \\
340 & Decrease & $070 \pm \Delta \varphi$ & Increase* $^{*}$ & $250 \pm \Delta \varphi$ \\
\hline
\end{tabular}

* Side of the planet where the significant changes were located.

equatorial radiation. These authors actually showed that the changes in the appearance of Jupiter's radiation belts can be strongly related to $D_{\mathrm{E}}$ and to the warp of the magnetic equator when the geometric parameter is varying by a few degrees. For the VLA 1997 and 2002 campaigns, the values of $D_{\mathrm{E}}$ were essentially $0^{\circ}$. The fluctuations in the peak brightness distribution observed in 1997 and 2002 are too significant to be associated with the small variation of $D_{\mathrm{E}}$ during the two campaigns of VLA observations.

Our analysis of the VLA 2002 data sets demonstrated that the short-term variability was observed when the angular sectors covering the Jupiter SIII longitudes $\lambda_{\text {III }}$ where the field strength along the equatorial magnetic surface is maximum or minimum were monitored. New evidences of short-term variations of Jupiter's synchrotron emission are thus expected to be reported when very specific CMLs are observed.

Along the equatorial surface, the synchrotron-emitting electrons contributing to the maximum of the radiation intensity at $\lambda$-cm wavelength have the energy $E_{\mathrm{Imax}} \approx 112 /\left(\lambda \times B_{\mathrm{eq}}\right)^{1.5}-$ 0.511 (Carr et al. 1983; Santos-Costa 2001). In the dipolar approximation, this relation becomes $E_{\mathrm{Imax}} \approx 112 \times\left[L^{3 / 2} /(\lambda \times\right.$ $\left.\left.B_{0}\right)^{1.5}\right]-0.511$. The Jovian radiation-belt electrons with energies between 30 and $40 \mathrm{MeV}$ are thus the particles primarily responsible for the changes in brightness distribution observed at $6 \mathrm{~cm}$. Further, the peak brightness distribution near $1.4 R_{\mathrm{J}}$ is radiated by high-energy electrons populating a region close to the Jovian Halo's inner edge where the 2:1 Lorentz resonance is taking place (Ockert-Bell et al. 1999; Burns et al. 2004). Short-term variations of Jupiter's Decimetric brightness distribution may be the result of submicrometre charged dust particles undergoing significant electromagnetic perturbations, and consequently drastic changes in their density, while interacting with the radiation-belt electrons in the Jovian ring's innermost component.

Repeating the monitoring of Jupiter's synchrotron emission at multiple times spread out over a few months is crucial to validating our discovery of short-term variability. A new campaign of observations with the VLA has been proposed to provide additional two-dimensional images to analyze and compare with our maps constructed from the VLA 2002 Fall data sets. From new data sets, we will be able to further examine the regions where the fluctuations are generated and investigate the processes responsible for short-term variations. We plan on constraining our theoretical modeling of 2-D images of
Jupiter's radiation-belt emission (Santos-Costa \& Bolton 2008) with VLA observations in order to further discuss the mechanism responsible for short-term changes in Jupiter's Decimetric emission.

Acknowledgements. This work was performed at Southwest Research Institute and the University of Melbourne. This research was carried out under NASA grant NNG05GR39G.

\section{References}

Berge, G. L. 1966, ApJ, 146, 767

Berge, G. L. 1974, ApJ, 191, 775

Bolton, S. J. 1990, in Time Variability of Jupiter's Synchrotron Radiation, U.C. Berkeley Thesis

Bolton, S. J., Janssen, M., Thorne, R., et al. 2002, Nature, 415, 987

Bolton, S. J., et al. 2004, in Jupiter: The Planet, Satellites and Magnetosphere, ed. F. Bagenal et al., Chap. 27, 671

Branson, N. J. B. A. 1968, MNRAS, 139, 155

Burns, J. A., Simonelli, D. P., Showalter, M. R., et al. 2004, in Jupiter: The Planet, Satellites and Magnetosphere, ed. F. Bagenal et al., Chap. 11, 241

Carr, T. D., Desch, M. D., \& Alexander, J. K. 1983, in Physics of the Jovian Magnetosphere, ed. A. J. Dessler, 226

Connerney, J. E. P. 1993, J. Geophysical Res., 98 (E10), 18659

de Pater, I. 1980, A\&A, 88, 175

de Pater, I., \& Dickel, J. R. 1986, ApJ, 308, 459

de Pater, I., \& Dunn, D. E. 2003, Icarus, 163 (2), 449

de Pater, I., \& Jaffe, W. J. 1984, ApJS, 54, 405

de Pater, I., \& Klein, M. J. 1989, in Time-Variable Phenomena in the Jovian System, ed. Belton et al., NASA SP-494, 139

de Pater, I., Kenderline, S., \& Dickel, J. R. 1982, Icarus, 51, 25

Dessler, A. J. 1983, in Physics of the Jovian Magnetosphere, ed. A. J. Dessler, 498

Dulk, G. A., Leblanc, Y., Sault, R. J., Ladreiter, H. P., \& Connerney, J. E. P. 1997, A\&A, 319, 282

Dulk, G. A., Leblanc, Y., Sault, R. J., \& Bolton, S. J. 1999, A\&A, 347, 1039

Galopeau, P. H., \& Gerard, E. 2001, Planet. Space Science, 49, 1379

Gerard, E. 1970, Radio Sci. J., 5, 513

Gerard, E. 1976, A\&A, 50, 353

Gulkis, S., Gary, B., Klein, M. J., \& Stelzried, C. T. 1973, Icarus, 18, 181

Khurana, K. K., et al. 2004, in Jupiter: The Planet, Satellites and Magnetosphere, ed. F. Bagenal, et al., Chap. 24, 593

Klein, M. J. 1976, J. Geophys. Res., 81, 3380

Klein, M. J., Gulkis, S., \& Stelzried, C. T. 1972, ApJ, 176, L85

Klein, M. J., Thompson, T. J., \& Bolton, S. J. 1989, in Time-Variable Phenomena in the Jovian System, ed. Belton et al., NASA SP-494, 151

Klein, M. J., Bolton, S. J., Gulkis, S., et al. 2001, in Planetary and Radio Emission, 221

Kloosterman, J. L., Buttler, B., \& de Pater, I. 2008, Icarus, 193, 644

Leblanc, Y., Dulk, G. A., Sault, R. J., \& Hunstead, R. W. 1997, A\&A, 319, 274

Mihoshi, Y., Misawa, H., Morioka, A., et al. 1999, Geophys. Res. Lett., 26, 9

Morris, D., \& Berge, G. L. 1962, ApJ, 136, 286

NRAO Green Book 1992, in An Introduction of the NRAO Very Large Array, ed. R. M. Hjellming, NRAO Publ.

Ockert-Bell, M. E., Burns, J. A., Daubar, I. J., et al. 1999, Icarus, 138, 188

Perley, R. A., \& Taylor, G. B. 2003, in The VLA Calibrator Manual, NRAO Publ.

Radhakrishnan, V., \& Roberts, J. A. 1960, Phys. Rev. Lett., 4, 493

Roberts, J. A., \& Huguenin, G. R. 1962, in La Physique des Planètes, Mem. Soc. R. Sci., 7, 587

Santos-Costa, D. 2001, in Modélisation des Ceintures de Radiation d'Electrons de Jupiter Internes à Io, ENSAE Ph.D. report

Santos-Costa, D., \& Bolton, S. J. 2008, Planet. Space Science, 56, 326

Sault, R. J., Teuben, P. J., \& Wright, M. C. H. 1995, in Astronomical Data Analysis Software and Systems IV, ASP Conf. Ser., 77, 433

Sault, R. J., Oosterloo, T., Dulk, G. A., \& Leblanc, Y. 1997, A\&A, 324, 1190

Tsuchiya, F., Misawa, H., Imai, K., et al. 2009, Advances in Geosciences, in press 